\title{
Role of heating and current-induced forces in the stability of atomic wires
}

\author{
Z. Yang* \\ Surface Physics Laboratory (National Key Laboratory), Fudan University, Shanghai, 200433, China \\ M. Chshiev \\ Department of Physics, Virginia Polytechnic Institute and State University, Blacksburg, Virginia 24061-0435, USA
}

M. Zwolak

Physics Department, California Institute of Technology, Pasadena, California 91125, USA

Y.-C. Chen

Department of Physics, University of California, San Diego, La Jolla, California 92093-0319, USA

\author{
M. Di Ventra ${ }^{\dagger}$ \\ Department of Physics, University of California, San Diego, 9500 Gilman Drive, La Jolla, California 92093-0319, USA
}

(Received 16 November 2004; published 13 January 2005)

\begin{abstract}
We investigate the role of local heating and forces on ions in the stability of current-carrying aluminum wires. For a given bias, we find that heating increases with wire length due to a redshift of the frequency spectrum. Nevertheless, the local temperature of the wire is relatively low for a wide range of biases provided good thermal contact exists between the wire and the bulk electrodes. On the contrary, current-induced forces increase substantially as a function of bias and reach bond-breaking values at about $1 \mathrm{~V}$. These results suggest that local heating promotes low-bias instabilities if dissipation into the bulk electrodes is not efficient, while current-induced forces are mainly responsible for the wire breakup at large biases. We compare these results to experimental observations.
\end{abstract}

DOI: 10.1103/PhysRevB.71.041402

PACS number(s): 73.40.Jn, 73.40.Cg, 73.40.Gk, 85.65.+h

Atomic wires are an ideal testbed to study transport properties at the nanoscale. ${ }^{1,2}$ Physical phenomena that have been investigated in these systems include their quantized conductance, ${ }^{3}$ conductance and noise oscillations as a function of the wire length, ${ }^{4-7}$ heating, ${ }^{1,8,9}$ and current-induced atomic motion. ${ }^{8,10-12}$ The latter two properties, in particular, are of key importance in understanding the stability of atomic wires under current flow. ${ }^{12}$

Halbritter et al. ${ }^{13}$ and, more recently, Mizobata et al. ${ }^{14,15}$ have explored the mechanical stability of atom-sized $\mathrm{Al}$ wires. These authors found that the probability of forming single-atom contacts decreases with increasing bias and that it vanishes at a critical bias of about $1 \mathrm{~V}^{16,17}$ In addition, several samples exhibited low-bias instabilities (typically at biases of $100 \mathrm{mV}$ or less) leading to breakup of the atomic wires. Similar trends have been reported in the case of $\mathrm{Pb}$ (Ref. 13) and Au (Ref. 4) point contacts, suggesting that the mechanisms leading to such instabilities are material independent. These low-bias instabilities have typically been attributed to inadequate dissipation of heat into the bulk electrodes. ${ }^{4,8,9}$ However, additional forces on ions due to current flow may also contribute, since both effects are present at any given bias.

In this paper, we explore the relative role of local heating and current-induced forces in the stability of aluminum wires at different biases. We study these effects perturbatively, i.e., we first calculate current-induced forces on ions while neglecting local heating; second, we assume current-induced forces are negligible and evaluate the local temperature of the wires. This perturbative approach is supported a posteriori: we find that local heating is substantial at very low biases if poor thermal contacts exist between the wire and the bulk electrodes, ${ }^{8,9,18}$ while current-induced forces (in particular, the average force per atom, see below) are small at low biases but increase substantially with increasing external voltage. In addition, if the heat in the wire can be dissipated efficiently into the electrodes, the local temperature in the junction will be quite low at biases for which currentinduced forces reach bond-breaking values. These results suggest that low-bias instabilities of atomic wires, if any, are due to local heating while high-bias breakup of a junction is mainly due to current-induced forces.

A schematic of one of the wires investigated is depicted in the inset of Fig. 1(a). It consists of an Al atomic chain (the inset shows a one-atom wire) sandwiched between two Al electrodes that we model with ideal metals (jellium model, $\left.r_{\mathrm{s}} \approx 2\right) .{ }^{19-21}$ The stationary scattering wave functions of the whole system are calculated by solving the LippmannSchwinger equation iteratively to self-consistency. Exchange and correlation are included in the density-functional formalism within the local-density approximation. ${ }^{19-21}$ More details of the calculations of current, forces, and heating can be found in the original papers..$^{9,12,21,22}$

We investigate the transport properties of $\mathrm{Al}$ chains with up to four atoms. All atomic coordinates have been relaxed to the equilibrium value at zero bias. ${ }^{23}$ The relaxed Aljellium edge bond length is about 2.0 a.u. and the relaxed $\mathrm{Al}-\mathrm{Al}$ bond distance decreases from 6.8 a.u. in the two-Al wire to about 5.8 a.u. in the four-Al wire. The bond lengths in longer wires are shorter and approach the bulk bond length, due to the reduced interaction of the middle atoms with the electrodes. 


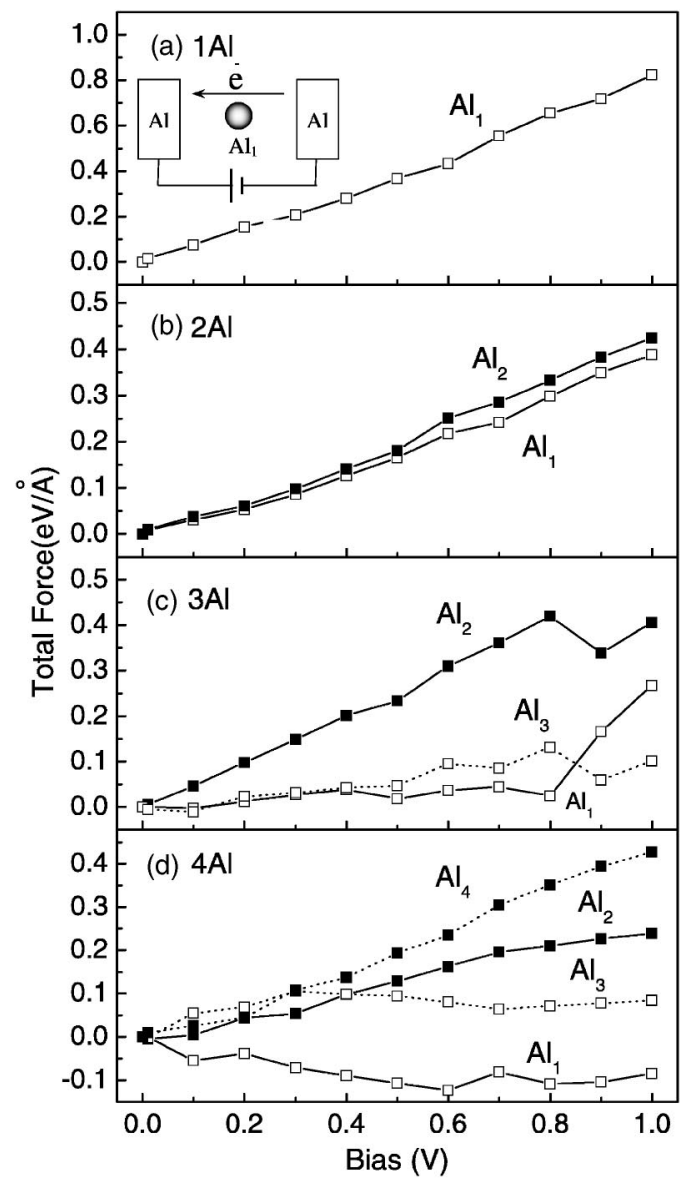

FIG. 1. Total current-induced forces as a function of bias in atomic wires containing (a) one, (b) two, (c) three, and (d) four atoms. The inset shows a schematic of one of the wires investigated. The atoms are labeled from the left electrode. The left electrode is positively biased. Positive force pushes the atom against electron flow.

Current-induced forces. The force on an ion, with position $\mathbf{R}$, due to current flow is calculated as ${ }^{12,22,24}$

$$
\mathbf{F}=-\sum_{i}\left\langle\psi_{i}\left|\frac{\partial v}{\partial \mathbf{R}}\right| \psi_{i}\right\rangle-\lim _{\Delta \rightarrow 0} \int_{\sigma} d E\left\langle\psi_{\Delta}\left|\frac{\partial v}{\partial \mathbf{R}}\right| \psi_{\Delta}\right\rangle,
$$

where $v$ is the electron-ion interaction. The first term on the RHS of Eq. (1) is similar to the usual Hellmann-Feynman contribution to the force due to localized electronic states $\left|\psi_{i}\right\rangle$. The second term is the contribution due to continuum states. $^{22}$ The wave functions $\left|\psi_{\Delta}\right\rangle$ are eigendifferentials for each energy interval $\Delta$ in the continuum $\sigma .^{22}$ Finally, the forces due to ion-ion interactions are added to obtain the total force. The total current-induced forces as a function of applied bias in $\mathrm{Al}$ wires containing one, two, three, and four atoms are shown in Figs. 1(a)-1(d), respectively. The atoms in the chains are denoted as $\mathrm{Al}_{i}(i=1, \ldots, 4)$ starting from the left electrode. Positive force indicates atoms are pushed to the right, i.e., against the electron flow. For the small external bias of $0.01 \mathrm{~V}$, the forces on $\mathrm{Al}_{1}$ and $\mathrm{Al}_{2}$ in the two- $\mathrm{Al}$ wire are in the same direction and have almost equal values [see

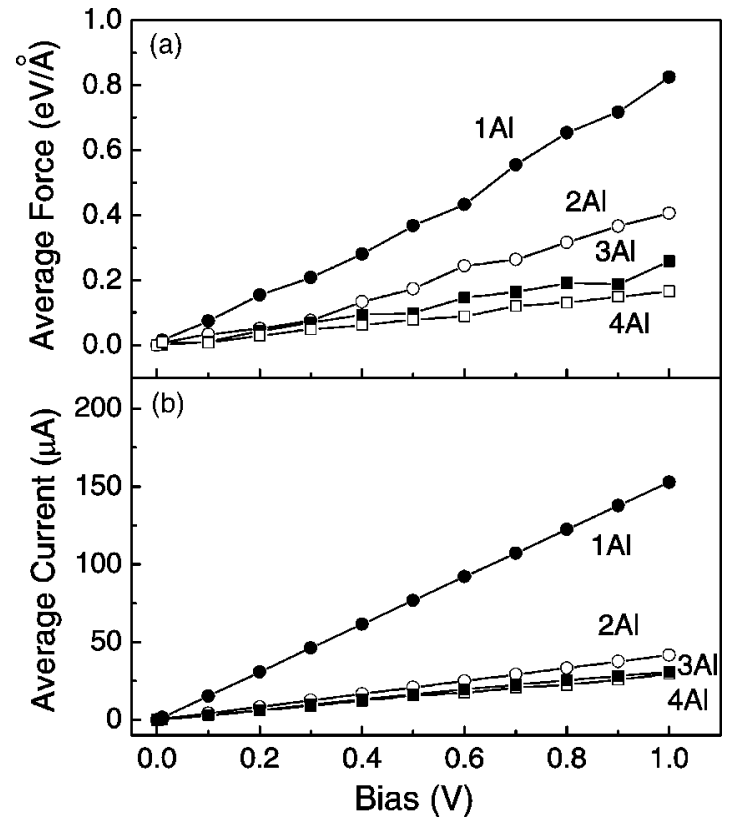

FIG. 2. (a) Average force on ions as a function of bias for Al wires of different length. (b) Total current divided by the number of atoms in the wire as a function of bias.

Fig. 1(b)], indicating the symmetry of the two atoms. For three-Al and four-Al wires, the forces obey the zero-sum rule in the limit of zero bias, as also found in Refs. 10 and 25: the force on $\mathrm{Al}_{2}$ is opposite to that of $\mathrm{Al}_{1}$ and $\mathrm{Al}_{3}$ in the three- $\mathrm{Al}$ wire, and the ones on $\mathrm{Al}_{1}$ and $\mathrm{Al}_{4}$ are opposite to those of $\mathrm{Al}_{2}$ and $\mathrm{Al}_{3}$ in the four-Al wire, and the sum of the forces of each wire is close to zero.

For one-Al and two-Al wires current-induced forces move the atoms against the electron flow at any bias (see Fig. 1) and increase quite linearly with increasing voltage up to $1 \mathrm{~V}$. On the other hand, for the three-Al and four-Al wire cases, the forces on ions are nonlinear at small voltages with some atoms moving against and some along with the current flow. The direction and magnitude of these forces are related to the local resistivity dipoles that form around the atoms. ${ }^{10}$

Despite these nonlinearities as a function of bias, the average force per atom follows a simple trend: it increases almost linearly with bias and, for a given external voltage, it decreases with wire length [see Fig. 2(a)]. Similar results have been reported for $\mathrm{Si}$ atomic chains. ${ }^{10}$ This trend correlates well with the corresponding total current divided by the number of atoms in the wire as a function of bias [Fig. 2(b)]. The figure shows that both the current divided by the number of atoms and the force saturate with length. This is consistent with the fact that (i) the wire has no defects and the resistance is mainly determined by the contacts, and (ii) the average force due to charge redistribution reaches a constant value with increasing wire length, since the stationary states become evenly distributed across the length of wire.

While it is difficult to predict the exact bias at which an atomic chain breaks due to current-induced forces, we can estimate it using total-energy calculations. For $\mathrm{Al}$ atomic wires, Jelínek et al. have found by first-principles calculations that the maximum force the wires can stand is about 0.6 


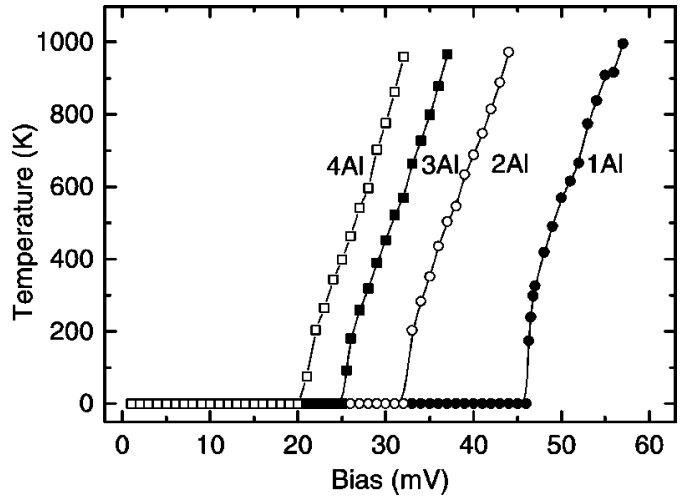

FIG. 3. Local temperature as a function of bias for $\mathrm{Al}$ wires of different lengths. No heat dissipation into the bulk electrodes is taken into account.

eV/Å. ${ }^{26}$ From Fig. 2(a) such force corresponds to a bias of about $0.8 \mathrm{~V}$. This value compares very well with the reported experimental result of $0.8 \mathrm{~V}$ by Mizobata et al. for singleatom $\mathrm{Al}$ contacts. ${ }^{14,15} \mathrm{It}$ is also evident that, on average and everything else being equal, larger voltages are required to break longer wires [Fig. 2(a)]. However, the breakup of the wires at high bias is likely to nucleate from the bonds of the atom with the largest force.

Local heating. We now explore the effect of heating assuming current-induced forces are zero. Heating occurs when electrons exchange energy with the lattice via absorption and emission of vibrational modes. Details of the theory of local heating in nanoscale structures can be found in Refs. 9 and 27. Here we just mention that there are two major processes that lead to a given local temperature in a nanojunction. One is due to inelastic processes that occur in the region of the junction. In this case electrons incident from the right or left electrode can absorb (cooling) or emit (heating) energy because of electron-vibration scattering in the junction. The other is due to dissipation of energy into the bulk electrodes. Let us first focus on the inelastic scattering contribution assuming the dissipation into the electrodes is negligible. This can be the result of, e.g., weak coupling of vibrational modes localized in the junction with the continuum of modes of the bulk electrodes. ${ }^{27}$

Denoting by $W_{v}^{L(R), 1(2)}$ the power absorbed (emitted) by electrons incident from the left (right) via a vibrational mode $\nu$, the total thermal power generated in the junction can be written as the sum over all vibrational modes of four scattering processes, ${ }^{9}$

$$
P=\sum_{\nu \in v i b}\left(W_{\nu}^{R, 2}+W_{\nu}^{L, 2}-W_{\nu}^{R, 1}-W_{\nu}^{L, 1}\right) .
$$

This power can be expressed in terms of the electronvibrational coupling in the presence of current. This coupling, in turn, can be determined from the same stationary scattering wave functions used to calculate forces on the ions. ${ }^{9}$ When the heating processes $\left(W_{\nu}^{R, 2}\right.$ and $\left.W_{\nu}^{L, 2}\right)$ balance the cooling processes $\left(W_{\nu}^{R, 1}\right.$ and $\left.W_{v}^{L, 1}\right)$, i.e., $P=0$, a steady-

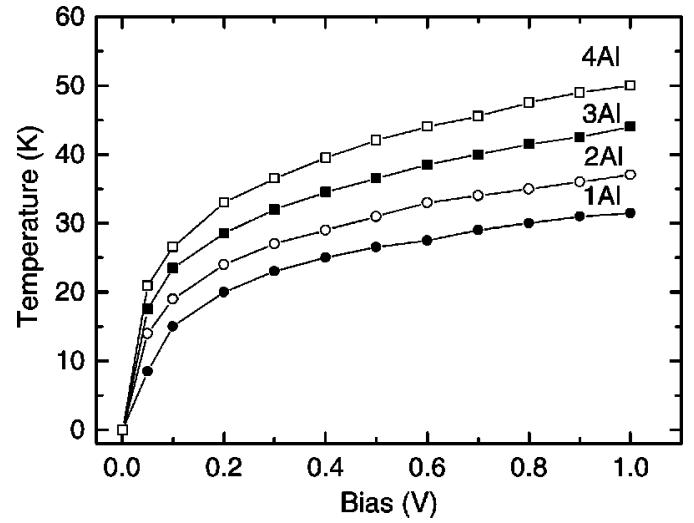

FIG. 4. Local temperature as a function of bias for $\mathrm{Al}$ wires of different lengths. Heat dissipation into the bulk electrodes is taken into account.

state local temperature is established in the junction. This temperature is plotted in Fig. 3 assuming zero background temperature (the same temperature is assumed throughout this paper).

A bias larger than the first vibrational frequency of the junction is necessary to generate heat (see Fig. 3).$^{28}$ One can see that the equilibrium temperature increases abruptly above that threshold bias and becomes substantial at biases of only a few $\mathrm{mV}$ (Fig. 3). This implies that if the heat generated in the junction is not efficiently dissipated into the electrodes, very large local temperatures will be generated even at low biases; thus inducing structural instabilities and consequent wire breakup. These instabilities have indeed been observed in Al wires, ${ }^{13-15}$ as well as in other metallic wires. ${ }^{4,29}$ Due to a redshift of the frequency spectrum with increase of wire length at a given bias, longer wires have substantially larger local temperatures. Note that at these same biases, currentinduced forces are negligible (see Fig. 1). However, if there is sufficient coupling between the junction vibrational modes and the modes of the bulk electrodes, this will allow the heat to dissipate from the junction into the bulk electrodes via elastic phonon scattering. ${ }^{9,30}$ This results in a much lower local temperature of the junction (see Fig. 4). It is clear that at the biases where current-induced forces reach bondbreaking values, the temperature in the junction is still quite low (less than about $50 \mathrm{~K}$ for all wires at $1 \mathrm{~V}$ ) with shorter wires having lower temperatures.

In conclusion, we find that both local heating and currentinduced forces can lead to structural instabilities, but at different ranges of the external bias. If there is poor thermal contact between the junction and the electrodes, local heating induces instabilities in the structure at small biases. At high biases, breakup of nanoscale junctions is mainly due to current-induced forces. These results help illuminate a very important aspect of nanoscale electronics.

Z.Y. acknowledges support from the NSF Grant No. 10304002 of China. M.D. acknowledges support from the NSF Grant No. DMR-01-33075. M.Z. acknowledges support from an NSF Graduate Fellowship. 
*Email address: zyang@fudan.edu.cn

${ }^{\dagger}$ Email address: diventra@ physics.ucsd.edu

${ }^{1}$ N. Agräit, A. Levy-Yeyati, and J. van Ruitenbeek, Phys. Rep. 377, 81 (2003).

${ }^{2}$ C. J. Muller, J. M. van Ruitenbeek, and L. J. de Jongh, Phys. Rev. Lett. 69, 140 (1992).

${ }^{3}$ J. C. Cuevas, A. Levy Yeyati, A. Martin-Rodero, G. Rubio Bollinger, C. Untiedt, and N. Agraït, Phys. Rev. Lett. 81, 2990 (1998).

${ }^{4}$ R. H. M. Smit, C. Untiedt, G. Rubio-Bollinger, R. C. Segers, and J. M. van Ruitenbeek, Phys. Rev. Lett. 91, 076805 (2003).

${ }^{5}$ K. S. Thygesen and K. W. Jacobsen, Phys. Rev. Lett. 91, 146801 (2003).

${ }^{6}$ N. D. Lang and Ph. Avouris, Phys. Rev. Lett. 81, 3515 (1998); N. D. Lang, ibid. 79, 1357 (1997).

${ }^{7}$ Y.-C. Chen and M. Di Ventra, Phys. Rev. B 67, 153304 (2003).

${ }^{8}$ T. N. Todorov, J. Hoekstra, and A. P. Sutton, Phys. Rev. Lett. 86, 3606 (2001).

${ }^{9}$ Y.-C Chen, M. Zwolak, and M. Di Ventra, Nano Lett. 3, 1691 (2003); 4, 1709 (2004).

${ }^{10}$ Z. Yang and M. Di Ventra, Phys. Rev. B 67, 161311(R) (2003).

${ }^{11}$ G. Rubio, N. Agrait, and S. Vieira, Phys. Rev. Lett. 76, 2302 (1996).

${ }^{12}$ M. Di Ventra, Y.-C. Chen, and T. N. Todorov, Phys. Rev. Lett. 92, 176803 (2004).

${ }^{13}$ A. Halbritter, Sz. Csonka, O. Yu. Kolesnychenko, G. Mihály, O. I. Shklyarevskii, and H. van Kempen, Phys. Rev. B 65, 045413 (2002).

${ }^{14}$ J. Mizobata, A. Fujii, S. Kurokawa, and A. Sakai, Jpn. J. Appl. Phys., Part 1 42, 4680 (2003).

${ }^{15}$ J.-I Mizobata, A. Fujii, S. Kurokawa, and A. Sakai, Phys. Rev. B 68, 155428 (2003).

${ }^{16}$ A. I. Yanson, G. Rubio-Bollinger, H. E. van den Brom, N. Agraït, and J. M. Van Buitenbeek, Nature (London) 395, 783 (1998).

${ }^{17}$ H. Yasuda and A. Sakai, Phys. Rev. B 56, 1069 (1997); K. Itakura, K. Yuki, S. Kurokawa, H. Yasuda, and A. Sakai, ibid. 60, 11163 (1999).

${ }^{18}$ R. H. M. Smit, C. Untiedt, and J. M. van Ruitenbeek, Nanotechnology 15, S472 (2004).

${ }^{19}$ N. D. Lang, Phys. Rev. B 52, 5335 (1995).
${ }^{20}$ N. D. Lang, Phys. Rev. B 49, 2067 (1994); Z. Yang, A. Tackett, and M. Di Ventra, ibid. 66, 041405(R) (2002).

${ }^{21}$ M. Di Ventra and N. D. Lang, Phys. Rev. B 65, 045402 (2002).

${ }^{22}$ M. Di Ventra and S. T. Pantelides, Phys. Rev. B 61, 16207 (2000).

${ }^{23}$ The initial Al-Al and Al-jellium edge distances have been chosen to be 5.4 and 2.6 a.u., respectively, (see Ref. 19).

${ }^{24}$ T. N. Todorov, J. Phys.: Condens. Matter 13, 10125 (2001); 14, 3049 (2002).

${ }^{25}$ T. N. Todorov, J. Hoekstra, and A. P. Sutton, Philos. Mag. B 80, 421 (2000).

${ }^{26}$ P. Jelínek, R. Pérez, J. Ortega, and F. Flores, Phys. Rev. B 68, 085403 (2003).

${ }^{27}$ M. J. Montgomery, T. N. Todorov, and A. P. Sutton, J. Phys.: Condens. Matter 14, 5377 (2002).

${ }^{28}$ We have employed Hartree-Fock total-energy calculations [see, e.g., J. A. Boatz and M. S. Gordon, J. Phys. Chem. 93, 1819 (1989)] to evaluate the vibrational modes of the atomic junctions. The surface geometry is (111) represented by a triangular pad of $\mathrm{Al}$ atoms with infinite mass. The force constants of the linear Al chains between the two pads are calculated first. Then, assuming only longitudinal motion of the $\mathrm{Al}$ atoms, the vibrational modes are determined.

${ }^{29}$ A. Fujii, J. Mizobata, S. Kurokawa, and A. Sakai, e-J. Surf. Sci. Nanotech. 2, 125 (2004).

${ }^{30} \mathrm{We}$ estimate the thermal conductance following the approach of K. R. Patton and M. R. Geller [Phys. Rev. B 64, 155320 (2001)]. We assume the junction to be a weak thermal link with a given stiffness $K$, which we evaluate from the frequency of the first vibrational mode of each structure. The values of the stiffness are $1.93,1.93,1.76$, and $1.59 \mathrm{eV} / a_{0}^{2}$ for wires of one, two, three, and four atoms, respectively. $a_{0}$ is the Bohr radius. We then estimate the thermal current into the electrodes via elastic phonon scattering as

$$
I_{t h}=\frac{4 \pi K^{2}}{\hbar} \int d \varepsilon \varepsilon N_{L}(\varepsilon) N_{R}(\varepsilon)\left[n_{L}(\varepsilon)-n_{R}(\varepsilon)\right],
$$

where $n_{L(R)}$ is the Bose-Einstein distribution function and $N_{L(R)}(\varepsilon)$ is the spectral density of states at the left (right) electrode surface. 